\title{
Selection, Visualization, and Interpretation of Deep Features in Lung Adenocarcinoma and Squamous Cell Carcinoma
}

Taher Dehkharghanian, ${ }^{* \dagger}$ Shahryar Rahnamayan, ${ }^{*}$ Abtin Riasatian, ${ }^{\ddagger}$ Azam A. Bidgoli, ${ }^{*}$ Shivam Kalra, ${ }^{\ddagger}$ Manit Zaveri, Morteza Babaie, ${ }^{\ddagger}$ Mahjabin S. Seyed Sajadi, Ricardo Gonzalelz, ${ }^{\ddagger}$ Phedias Diamandis, ${ }^{\ddagger}$ Liron Pantanowitz, "Tao Huang, " and Hamid R. Tizhoosh

From the Nature Inspired Computer Intelligence (NICI) Lab, * Ontario Tech University, Oshawa, Ontario, Canada; the Department of Pathology and Molecular Medicine, ${ }^{\dagger}$ McMaster University, Hamilton, Ontario, Canada; the KIMIA (Laboratory for Knowledge Inference in Medical Image Analysis) Lab, ${ }^{\ddagger}$ University of Waterloo, Waterloo, Ontario, Canada; the Department of Laboratory Medicine and Pathobiology, ${ }^{\S}$ University of Toronto, Toronto, Ontario, Canada; and the Department of Pathology, ${ }^{\circledR}$ University of Michigan, Ann Arbor, Michigan

Accepted for publication August 20, 2021.

Address correspondence to Hamid R. Tizhoosh, Ph.D., KIMIA Lab, University of Waterloo, 200 University Ave. W., Waterloo, ON, Canada, N2L 3G1. E-mail: hamid. tizhoosh@uwaterloo.ca.

\begin{abstract}
Although deep learning networks applied to digital images have shown impressive results for many pathology-related tasks, their black-box approach and limitation in terms of interpretability are significant obstacles for their widespread clinical utility. This study investigates the visualization of deep features (DFs) to characterize two lung cancer subtypes, adenocarcinoma and squamous cell carcinoma. It demonstrates that a subset of DFs, called prominent DFs, can accurately distinguish these two cancer subtypes. Visualization of such individual DFs allows for a better understanding of histopathologic patterns at both the whole-slide and patch levels, and discrimination of these cancer types. These DFs were visualized at the whole slide image level through DF-specific heatmaps and at tissue patch level through the generation of activation maps. In addition, these prominent DFs can distinguish carcinomas of organs other than the lung. This framework may serve as a platform for evaluating the interpretability of any deep network for diagnostic decision making. (Am J Pathol 2021, 191: 2172-2183; https:// doi.org/10.1016/j.ajpath.2021.08.013)
\end{abstract}

The histopathologic examination of human specimens using microscopes has been accepted as the gold standard for diagnosis, sometimes rendering prognosis, or discovery. However, with the rise of digital pathology, tissue samples can now be viewed on computer screens as whole slide images (WSIs), generated by digitizing glass slides using high-resolution scanners. ${ }^{1}$ Given the advances in digital pathology and the increasing availability of large-scale public data sets, histopathology has become a significant research area for applications of data-driven models, such as deep neural networks (DNNs). Deep learning has been implemented for tasks like image classification, ${ }^{2-4}$ object detection, $^{5,6}$ and tissue segmentation, among others. ${ }^{7-9}$ Deep networks have also been used for more complex tasks, such as genetic mutation prediction ${ }^{10}$ and survival analysis. $^{11}$ Furthermore, pretrained convolutional neural networks have been used for feature extraction for contentbased image retrieval. $^{12-15}$

The rising influence of artificial intelligence (AI) in health care, including pathology, has signified the importance of understanding how AI works for medical professionals. However, a survey of UK medical students showed that they do not consider themselves sufficiently prepared to utilize medical AI tools. ${ }^{16}$ Therefore, it is necessary for health care professionals and organizations to improve their capability to understand and accordingly better harness the power of

Supported by the Government of Ontario, Canada and the Ontario Research Fund-Research Excellence Gigapixel image identification consortium.

Disclosures: L.P. serves on the medical advisory board for Ibex and NTP and acts as a consultant for Hamamatsu. 
these emerging technologies. A better understanding of AI could increase trust and acceptance of AI technologies and help health care professionals utilize these tools more effectively in the medical fields. ${ }^{17-19}$

One of the core competencies that is required to understand how AI works is the ability to recognize and describe how AI systems make decisions. ${ }^{20} \mathrm{~A}$ significant issue with some current deep learning models is that their decision-making process is usually obscure from the end users (ie, the black box phenomenon). Furthermore, classification models are usually trained on large publicly available data sets. However, in pathology, these data sets are unlikely to be all inclusive for all tissues, disease types, and different specimen preparations (eg, frozen sections, formalin-fixed, paraffin-embedded sections, and hematoxylin and eosin stains). For example, The Cancer Genome Atlas (TCGA) program data set only contains WSIs of two lung cancer subtype ${ }^{21}$ [namely, lung adenocarcinoma (ADC) and lung squamous cell carcinoma (SCC)]. Hence, when developing an $\mathrm{AI}$ algorithm to recognize lung carcinoma by employing TCGA images, it is necessary to understand exactly what such an algorithm has learned.

These issues were the motivation behind exploring the decision-making process of KimiaNet, a DNN trained for cancer type classification that is aimed to be used for feature extraction. ${ }^{22}$ The KimiaNet network was selected for two reasons. First, access to TCGA data used for training and validating this DNN were readily available. Second, KimiaNet has been developed to serve as a domain-specific deep feature (DF) extractor for histopathologic images. These deep features could be used for content-based image retrieval and transfer learning, which is a common practice in applications of $\mathrm{AI}$ in histopathology..$^{23-25}$

For histopathology images, efforts have been made to make DNNs more interpretable through exploring deep features. The number of deep features are typically reduced to a subset containing important features. Schaumberg et $\mathrm{al}^{15}$ selected important deep features by shuffling deep feature values and calculating the effect of this shuffle on their model's classification accuracy. Faust et $\mathrm{al}^{26,27}$ showed that individual deep features of a DNN trained on brain tumor images are attributable to perceivable histomorphologic patterns. This property of deep features was investigated on deep feature activation maps. ${ }^{26,27}$ This study investigated each deep feature's classification capability independently from other deep features. The aim of this investigation was to evaluate the interpretability of the deep features generated by a trained DNN when applied to WSIs of lung cancers. This study has the potential to help domain experts who are not AI experts to evaluate models be used in their workflow.

\section{Materials and Methods}

KimiaNet DNN

KimiaNet $^{22}$ borrowed DenseNet's topology with four dense blocks $^{28}$ for the purpose of becoming a domain-specific feature extractor for histopathologic images. Technical details regarding KimiaNet development, validation, and application have been previously published. ${ }^{22}$ This network was trained to classify 30 cancer types based on TCGA data set using tissue patches of size $1000 \times 1000$ pixels at $\times 20$ magnification. KimiaNet achieved $86 \%$ accuracy when used for image search in lung cancer images of TCGA data.

\section{Digital Image Data Set}

Deep features (ie, output of the last pooling layer before the classification layer in the DNN) have been used in this study to classify lung cancer. KimiaNet's tissue patch sampling pipeline was applied to 829 diagnostic WSIs of lung ADC and lung SCC. These WSIs were collected from tissue samples of 759 patients. Patients with more than one diagnostic WSI were excluded from the following experiments, which resulted in a total of 735 WSIs from 403 SCC and 332 ADC patients. A total of 575 of these WSIs had already been used for training KimiaNet, 79 were used for validation, and 81 were spared for testing. There were 99,341 deep feature vectors of size 1024 for these 735 WSIs. Each WSI was represented by 135 patches on average, ranging from 2 to 617 patches per WSI. Benign regions in these lung images mostly consisted of alveolated lung parenchyma, perihilar fat, and cartilaginous airway, which are hypocellular when compared with regions displaying malignancy. Therefore, it is more likely that the training assumption of KimiaNet holds in lung cancer images. KimiaNet's training set was used for training models to distinguish ADC and SCC WSIs. Models were evaluated on KimiaNet's validation set, which had been seen by the network but had not been used for weight optimization. The test set images were spared for deep feature visualization in an attempt to understand what the network has learned during its training process.

It is well known that TCGA repository has several imperfections, manual markings and artifacts, missing pyramidal structures, and relatively low quality for frozen sections, among others. Diagnostic slides (formalin fixed, paraffin embedded) were selected with an aim to rely on a public data set for the sake of reproducibility of the results. TCGA lung images originated from a large number of hospitals, providing valuable samples for histologic diversity in lung cancer. All cases that entered TCGA database had to undergo a quality assurance process by experts to confirm the diagnosis. The reviewing pathologist (creator of the TCGA dataset) likely also had ancillary studies to confirm the diagnosis in challenging cases, assuring, the quality of the labeled data.

\section{Results}

\section{Median Deep Features for WSI Representation}

Since WSIs are often gigapixel images, computational and memory limitations make it almost impossible to feed them 


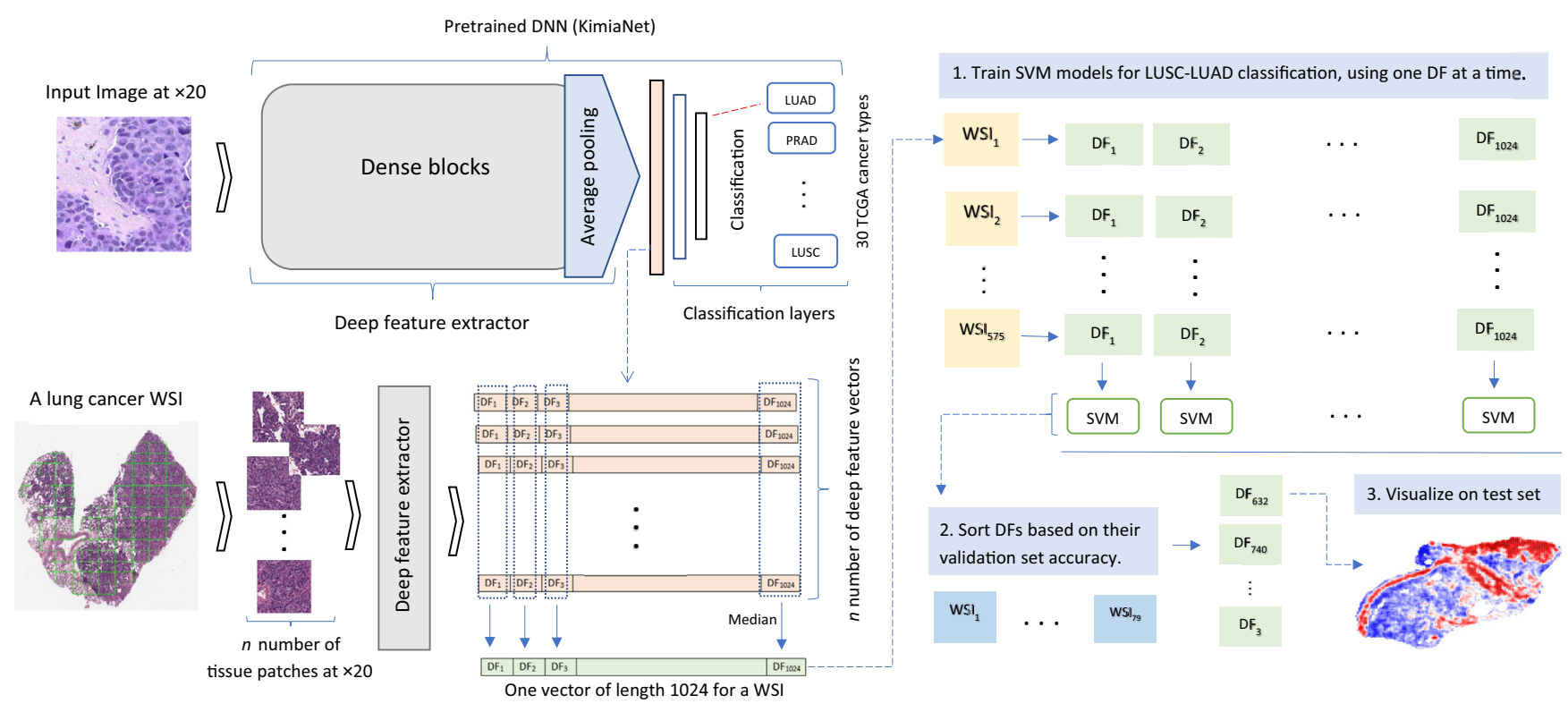

Figure 1 The process of generating vectors of median deep features (MDFs) for whole slide image (WSI) representation is illustrated. Tissue image patches were extracted from a WSI. The green colored grid on the WSI thumbnail shows the locations of selected tissue patches. Hypocellular patches were discarded. Retained patches were fed into KimiaNet to produce deep feature vectors. The MDF of each patch was calculated over all deep feature (DF) vectors. This process resulted in a single vector of length 1024 for each WSI. Support vector machine (SVM) classifiers were trained using only one deep feature for lung adenocarcinoma (LUAD) - lung squamous cell carcinoma (LUSC) classification. Deep features were sorted on the basis of their respective model classification accuracy. Deep features with the highest validation set accuracy became candidate deep features to be further investigated by visualization techniques on test WSIs. DNN, deep neural network; PRAD, prostate adenocarcinoma.

directly into convolutional neural networks. ${ }^{14}$ Therefore, smaller-sized samples should be taken out of different regions of a WSI to be fed to a pretrained DNN, to extract deep features that represent each sample's semantic content. The main objective was to find prominent deep features that play a major role in DNN's decision-making process. For lung WSIs, on average, there were 135 image patches for each WSI. Therefore, on average, feature extraction resulted in 135 deep feature vectors of length 1024 deep features for each WSI. Therefore, to find a simple representation for WSIs, median deep features (MDFs) were used across all patches of a WSI to generate one single feature vector for the entire specimen.

To determine if the MDF vector was a reliable WSI representation, MDF vectors were used for classifying lung ADC and lung SCC with a support vector machine (SVM) classifier. ${ }^{29}$ SVM algorithm is usually used for binary classification of pretrained features because of its simplicity and reliable performance compared with other models. ${ }^{30}$ However, other classifiers could also be used because the goal of this study is not to propose classifiers but to find prominent deep features. First, for each WSI, the MDF was calculated, which resulted in a single vector of length 1024 per WSI. This process is schematically described in Figure 1. Next, SVM with a linear kernel was trained to classify MDF vectors. SVM classifiers were trained on the original training set (575 samples). KimiaNet's validation set (79 samples) was reserved for model evaluation. The results showed the possibility of learning lung cancer subtypes from MDF with 91\% classification accuracy, as reported in Table 1.

MDF vectors effectively represented large WSIs for classification of lung ADC and SCC. This is mainly because the tissue patch selection pipeline in itself selected samples from areas with high cellularity, which are usually due to either the presence of malignant cells or the host's immune system response. Furthermore, TCGA requires diagnostic WSIs to be composed of at least $60 \%$ tumor nuclei (=https://www.ncbi.nlm.nih.gov/projects/gap/cgi-bin/study. cgi?study_id = phs000178.v1.p1_22, last accessed February $12,2021)$. Therefore, it is safe to assume that most selected patches were from malignant tissue regions. Using MDF vectors for WSI representation helped investigate each deep feature and find prominent deep features that could potentially correlate with lung cancer-related histopathologic patterns.

Table 1 Classification Performance Using MDF on KimiaNet's Validation Sample

\begin{tabular}{lllll}
\hline Type of cancer & Precision & Recall & F1-score & WSIs, N \\
\hline Adenocarcinoma & 0.97 & 0.84 & 0.90 & 38 \\
$\begin{array}{l}\text { Squamous cell } \\
\quad \text { carcinoma }\end{array}$ & 0.87 & 0.98 & 0.92 & 41 \\
Average/total & 0.92 & 0.91 & 0.91 & 79 \\
\hline
\end{tabular}

MDFs appear to be expressive WSI representation.

MDF, median deep feature; WSI, whole slide image. 


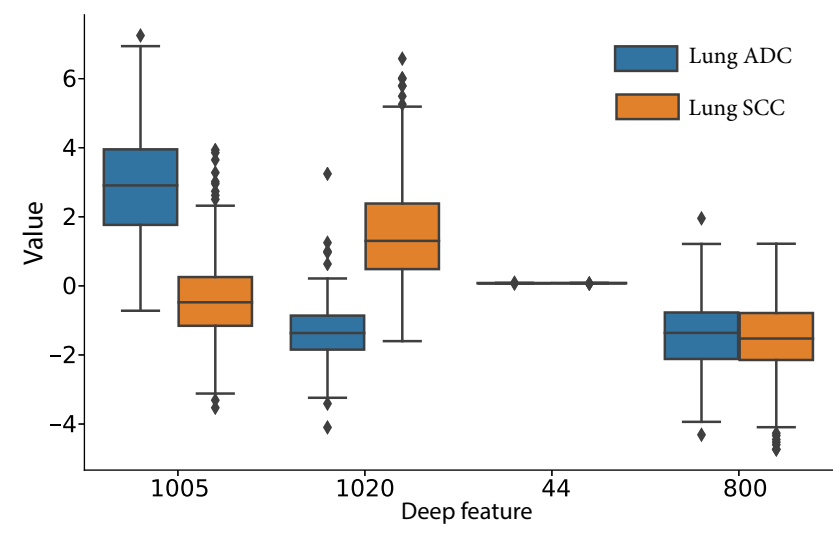

Figure 2 Division of median deep feature (DF) values into three main categories: Those that have high values among lung adenocarcinoma (ADC) or squamous cell carcinoma (SCC) cases (eg, DF 1005 and DF 1020), those with similar distributions among these two cancer types (eg, DF 800), and others features that do not represent either cancer subtype and accordingly have values near zero. The number shown after each DF represents its location on the deep feature vector.

\section{Deep Feature Exploration}

Deep features with significantly different distributions for lung ADC and lung SCC were observed (Figure 2). This finding suggests that a suite of deep features exists that could single handedly classify lung ADC and lung SCC with acceptable levels of accuracy. For human end users, this would immensely simplify the visualization and interpretation of deep features if expected to interact with an AI system. To find such interpretable deep features, onedimensional SVM classifiers (similar to defining a threshold) were trained on the training set, and evaluated on the validation set, as shown in Figure 1. Three of the deep features could distinguish lung ADC and SCC with $>90 \%$ accuracy. There were also 15 deep features that exhibited $>85 \%$ classification accuracy, as shown in Table 2. Also, two of the deep features could classify lung ADC and SCC as accurately as all 1024 features combined. Deep features with a classification accuracy of $>85 \%$ were selected for further examination. For the rest of this study, these 15 deep features are referred to as prominent deep features. A $t$-test was employed to see whether the difference in means of these prominent deep features among lung ADC and SCC groups was caused by chance. None of them had $P$ values $>0.001$. Finding these prominent deep features within MDFs that are notably accurate in distinguishing lung ADC and SCC provides an opportunity to attribute histologic patterns to each of them. Furthermore, as the number of selected features decreases, the model's interpretability increases.

\section{Deep Feature Visualization}

Having uncovered that there are some deep features whose median can single handedly distinguish lung ADC from SCC, it was necessary to subsequently resolve from which regions of a WSI these prominent deep features were originating. This would help visualize how these features may quantitatively and qualitatively differentiate tumor subtypes. To visualize deep features, tissue patches $(1000 \times 1000$ pixels) of WSIs were fed to KimiaNet, and deep feature vectors were extracted for each tissue patch. These feature vectors were used to generate heatmaps, which are color coded images where each pixel corresponds to the value a prominent deep feature at the location of tissue patches. To increase the resolution of heatmaps, tissue patches were selected with $66 \%$ overlaps. These heatmaps reveal from

Table 2 Lung ADC Versus SCC Classification Using Only One Deep Feature

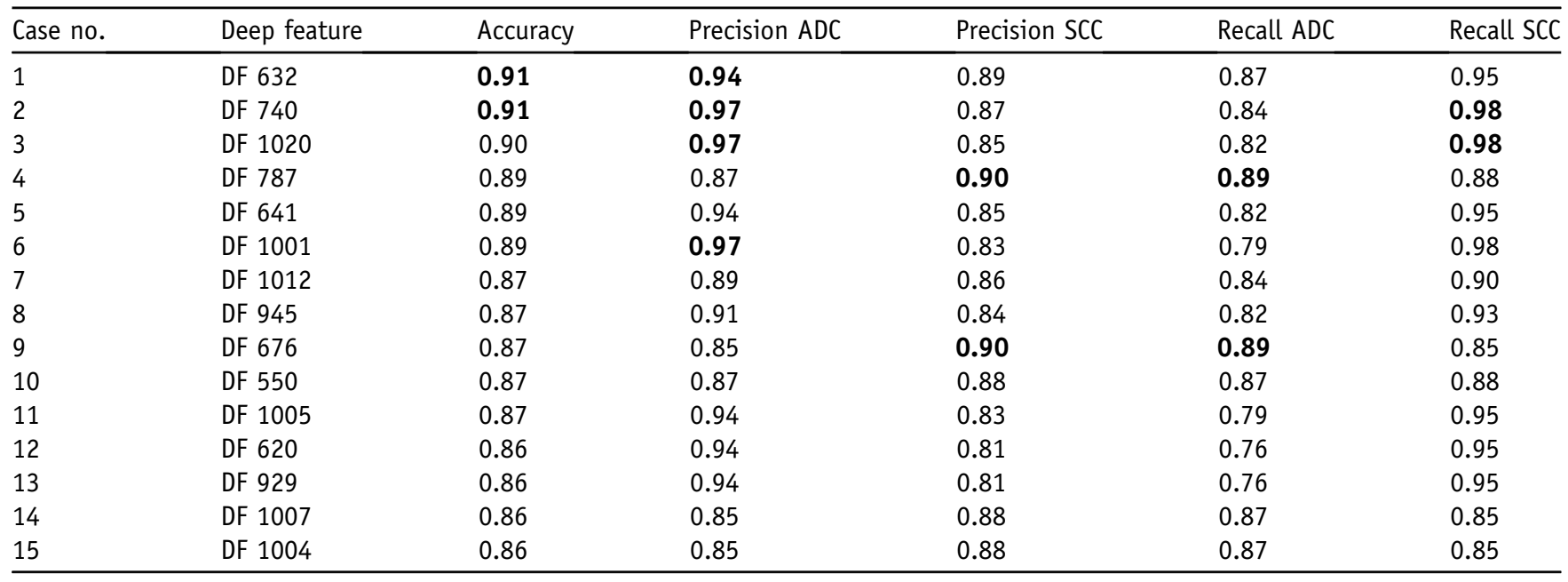

The top 15 DFs with the highest accuracy are shown, trained on KimiaNet's training set and evaluated on its validation set. There are three deep features (DF 632, DF 740, and DF 1020, among 1024 median DF values) that could single handedly distinguish lung ADC and SCC. The number shown after each DF represents its location on the DF vector. Highest values are marked in bold.

ADC, adenocarcinoma; DF, deep feature; SCC, squamous cell carcinoma. 

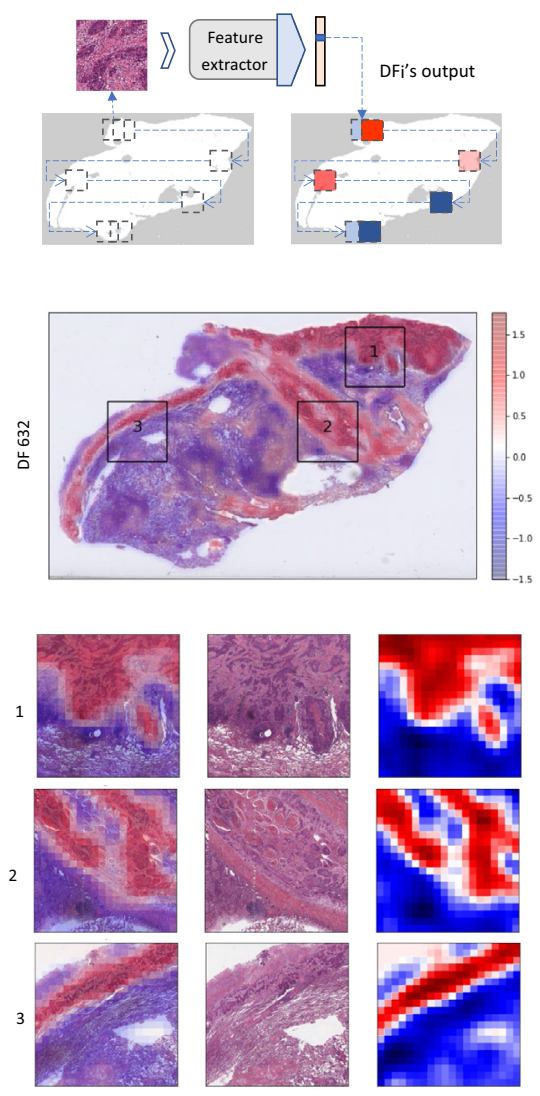
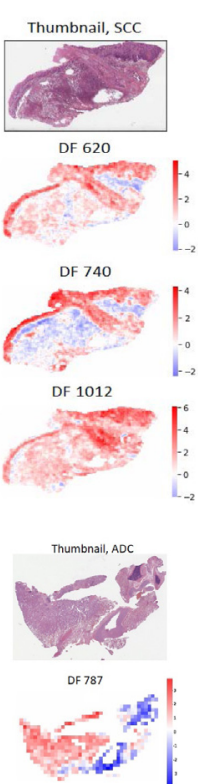

Figure heatmap's resolution. Color intensity represents cumulative deep feature value over a region. Numbers 1 to 3 show sample patches shown at a higher magnification. These images show that DF 632 signifies keratinizing lung SCC while exhibiting low values for keratin pearls. Middle: 0verlapping heatmap approach for five other deep features is shown. DF 1020 and DF 1012 strongly correspond to lung SCC. DF 1007 was the only feature that positively correlated to lung adenocarcinoma (ADC) that lacked glandular formation, whereas DF 929 and DF 1005 correlated to those differentiated lung ADCs that exhibited glandular formation. Right: Overlapping heatmaps of 14 deep features at the whole slide image (WSI) level; they reveal that these prominent deep features correlate with specific regions of WSIs that are visually similar. Tissue patches are $2000 \times 2000$ pixels at $\times 20$ magnification. However, their real size may differ as it is a factor of image resolution. Additional heatmaps are shown in Supplemental Figure S1.

which part of a WSI a discriminative feature value is emerging. A visual inspection showed that the prominent deep features were not haphazardly scaterred across the WSI but but corresponded to contiguous regions of WSIs (Figure 3).

These prominent lung cancer-related deep features could potentially be divided into three categories. The first category contains deep features that correlate to the concept of lung ADC and SCC rather than any particular visual pattern. In this case, the decision has been made upstream of these features/neurons. The second group contains those that could be attributed to visual histologic patterns that are prominent in either lung SCC or ADC. The last group is deep features that are irrelevant to histologic characteristics of lung cancer but represent the training data's internal biases, which happen to be useful for the classification of these two cancer types. None of the deep features have uniformly high or low values all over a WSI. Therefore, they do not seem to be histologically irrelevant features. This might suggest that these prominent deep features are less likely to correspond to the training data's internal biases.
The heatmap approach could be helpful in finding histopathologically meaningful features. One could easily localize spots with the highest value for a specific deep feature. Hence, deep features could be investigated at the WSI level rather than the patch level to find areas that correspond to a certain deep feature, which could serve as a simple alternative to attention models, which are visualization schemes to show areas within an input image with the highest significance on the model's output. ${ }^{31}$ Figure 3 shows that DF 632 strongly corresponds to keratinizing lung SCC while not being responsive to keratin pearls. DF 1012 and DF 1020 were also responsive to SSC. DF 1005 and DF 929 react to lung ADC with glandular features and not those with solid patterns. Through the investigation of heatmaps, it was found DF 1007 was the only deep feature among the prominent ones that corresponded with solid ADCs, in addition to other patterns. Sample heatmaps are provided in Supplemental Figure S1. In addition, deep features used in this study are the result of average pooling apply to the output of KimiaNet's last convolutional layer, of size $1024 \times 31 \times 31$ values. Therefore, it is easy to trace 

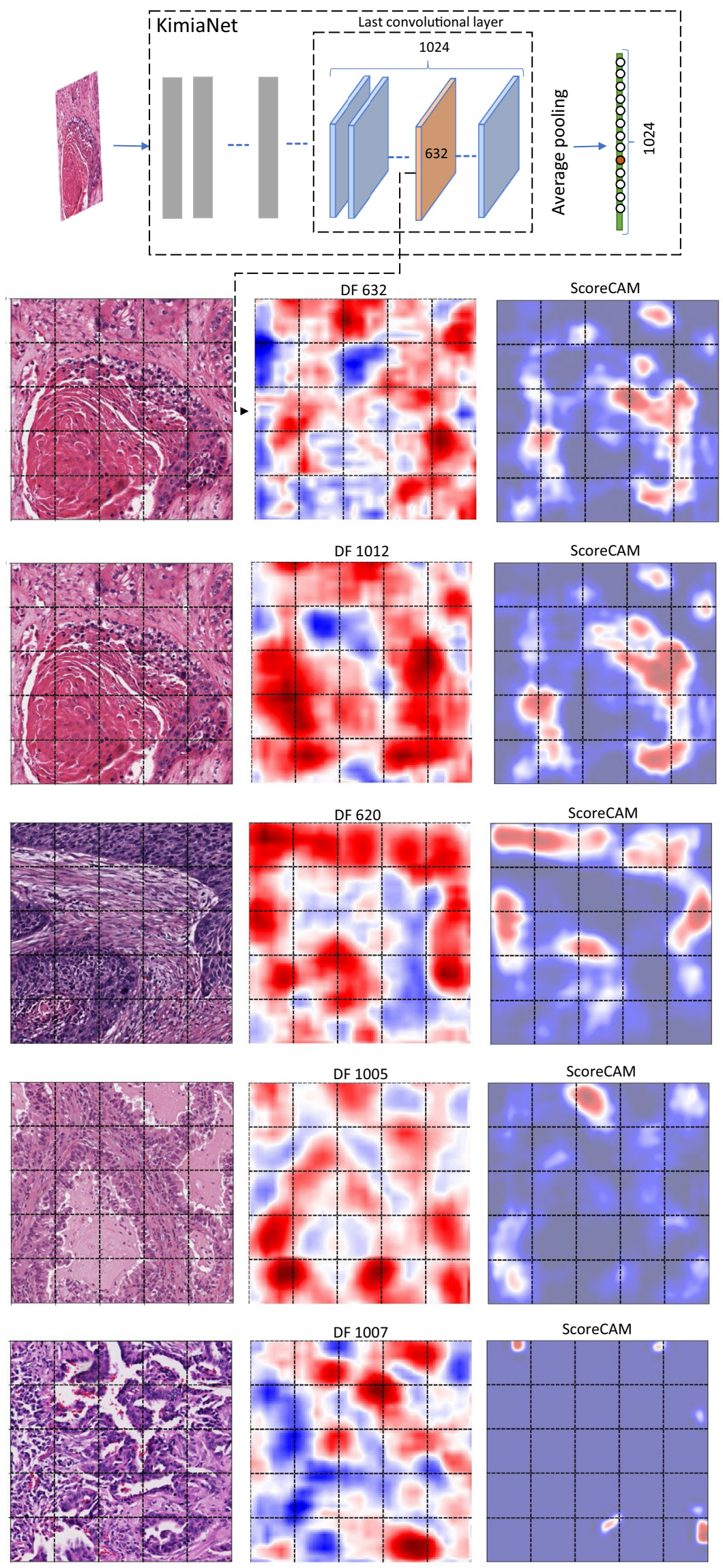

Figure 4 Heatmaps produced from the correspondence of convolutional layers and deep features (DFs). These maps were resized from $31 \times 31$ to $1000 \times 1000$ to have the same size as the tissue patches. ScoreCam maps have also been generated for these deep features and tissue patches. These deep features correspond well to malignant areas of a tissue patch and in some cases produce better results than ScoreCam. The black grid is added to images only to make localization easier for the viewer and has no other meaning. Tissue patches are $1000 \times 1000$ pixels at $\times 20$ magnification. However, their real size may differ as it is a factor of image resolution. 
back each deep feature to its corresponding $31 \times 31$ map and visualize it to check which regions of a patch have the highest intensity for any specific deep feature. These patchlevel heatmaps could serve as a simple alternative to class (feature) activation maps, which localize regions with the highest influence on the value of a feature.

These heatmaps correspond well to the malignant area, and it is shown that DF 632, in contrast to DF 1012, does not react to keratin pearls. They are also in congruence with ScoreCAM ${ }^{32}$ maps, and can even produce more meaningful results in some cases, like DF 1007 (Figure 4). ScoreCAM has a better performance compared with other similar DF visualization schemes, such as SmoothGrad, ${ }^{33}$ GradCAM,${ }^{34}$ and $\mathrm{GradCAM}++{ }^{35}$ Some examples are shown in Supplemental Figure S2.

\section{Generalization}

Ideally, deep features that work well for lung cancer classification should be useful for distinguishing other cancer types. If these 15 prominent deep features contain information that could be attributed to generally meaningful histologic patterns, they should also potentially be useful for ADC and SCC in nonpulmonary organs. For this experiment, TCGA WSIs were selected on the basis of their organ of origin and their primary diagnosis, and
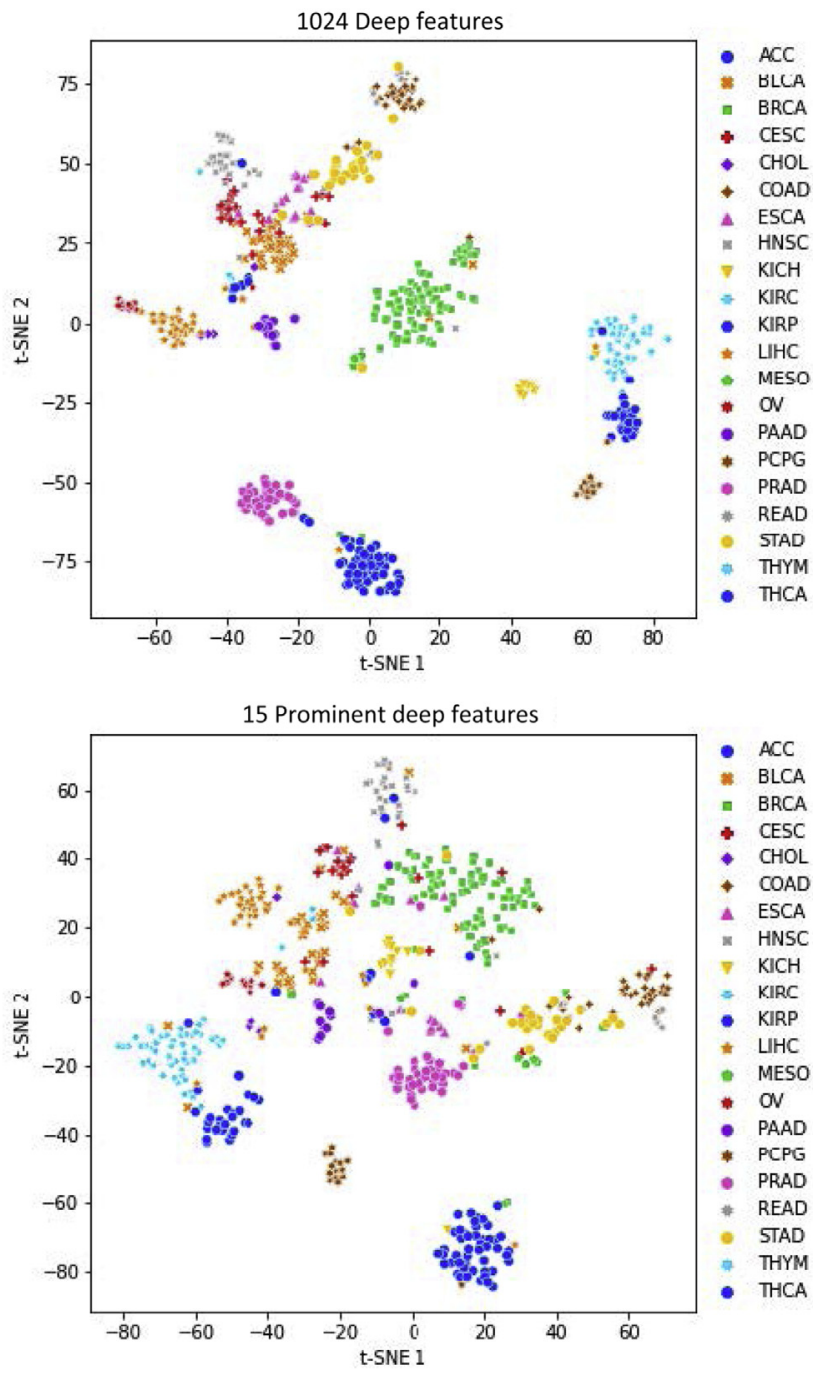

Figure 5 Left side: Hierarchical clustering of prominent lung cancer-related deep features shows that these deep features could be used to group carcinomas from nine nonpulmonary organs into meaningful clusters. Negative and positive values are represented by blue and orange, respectively. Colors intensify as the absolute value increases. Squamous cell carcinoma (SCC) and adenocarcinoma (ADC) cases are grouped in different clusters. Right side: The tdistributed stochastic neighbor embedding (t-SNE) distribution of 21 carcinomas from The Cancer Genome Atlas (TCGA) data set. Top: The plot was generated using medians of all 1024 deep features per whole slide image (WSI). Bottom: The plot was generated by using medians of 15 prominent lung cancer deep features. This shows that WSIs of a same cancer type group together relatively well even when using only 15 deep features. Therefore, lung cancer-related deep features contain information useful for distinguishing nonpulmonary cancer types. A list of abbreviations of TCGA's cancer types is provided in Supplemental Table S1. 
samples with primary diagnoses other than ADC and SCC were discarded. MDF vectors were generated for these WSIs of nine organs. These WSIs had not been used in KimiaNet's training phase. Hierarchical clustering method (the clustermap function from the seaborn python library ${ }^{36}$ ) was employed to group WSIs together based on their MDF values. Indeed, as shown in Figure 5, they were clustered into meaningful groups (a list of abbreviations is provided in Supplemental Table S1). All ADC cases were grouped together. The same occurred for SCC cases, except for a few cases of head and neck and esophageal squamous cell carcinomas. This finding suggests that these prominent deep features contain information pertaining to the visual characteristics of these two major carcinoma subtypes relatively independent of the primary tumor site.

It is also important to determine whether the aforementioned 15 prominent deep features identified on lung cancer subtypes could also generalize to carcinomas of other organs. MDF vectors were generated for WSIs of 21 nonpulmonary carcinomas that were included in TCGA data set. These WSIs had not been utilized for training the KimiaNet. Two t-distributed stochastic neighbor embedding plots were generated using all 1024 deep features and 15 lung cancer-related prominent deep features. These plots suggest that WSI of the same cancer type grouped together relatively well even when using only 15 deep features, as shown in Figure 5. This finding shows a promise to decrease the length of deep feature vectors by finding prominent organ-specific features that could be useful for carcinomas of other organs. Of note, these tdistributed stochastic neighbor embedding plots also indicate that the MDF vectors of tissue patches extracted from high cellular regions could succinctly represent WSIs of cancer of other organs besides lung cancer. However, this finding does not necessarily mean that these prominent DFs correspond to the same histologic patterns across carcinomas of other organs.

\section{Subjective Evaluation}

The interpretability of deep feature-specific heatmaps was evaluated at both WSI level and tissue patch level. Eleven WSIs (six SCCs and five ADCs) of the test set with high histologic diversity were selected to generate heatmaps. To facilitate the WSI evaluation, heatmaps of deep features correlated to each cancer type were combined, resulting in an ADC-correlated and an SCC-correlated heatmap for each WSI. These heatmaps were examined by the three coauthoring pathologists (L.P., R.G., and T.H.) to answer the question how well these heatmaps correspond to the locations of malignant tissue. In all these 11 samples, ADCcorrelated heatmaps were ranked higher for ADC cases, and SCC-correlated heatmaps were ranked higher for SCC cases. However, some SCC-correlated heatmaps still showed higher intensities on malignant regions of $\mathrm{ADC}$
WSIs, although not being as precise as ADC-correlated heatmaps. This was not observed in SCC WSIs.

Because of the structure of KimiaNet and the location that deep features were taken from, it is possible to show the output of a convolution layer that is solely responsible for the value of each deep feature. These two-dimensional arrays can be displayed as heatmaps. These heatmaps can be helpful to estimate the reliability of a decision made by the model. For the next experiment, these heatmaps were examined by the three co-authoring pathologists (L.P., R.G., and T.H.) to answer the question whether they were meaningful, and whether the model's decision was correct. As displayed in Figure 6, the likelihood of the model's decision is significantly higher when the heatmaps are histopathologically sensible. Consequently, such heatmap visualizations could help pathologists to decide how reliable the output of a model is.

To find histologic patterns that correlate with these prominent deep features, tissue patches were divided into 25 smaller-sized patches. These smaller tissue patches were sorted on the basis of their average value for each prominent DF, limited to five patches per WSI. For each DF, the top 25 patches were turned into a mosaic, and it was examined by the co-authoring pathologists (L.P., R.G., T.H., and P.D.) to determine whether there were any visually recognizable patterns. Examples are shown in Supplemental Figure S3. On the basis of this evaluation, one could divide DFs into three main categories:

i) DFs that correspond well with a specific histologic pattern specific to either ADC or SCC. For instance, DF 620 and DF 632 have high values on regions with intercellular bridges. DF 550 has high correspondence with SCC patches that show keratinization. DF 641 and DF 1007 have the highest values for patches with acinar formations.

ii) DFs with high values for either ADC or SCC, without any histologic patterns attributable to them. DF 787, DF 929, DF 945, DF 1001, and DF 1005 correlate to several patterns that can be seen in ADC samples. The same can be said for DF 1004 and DF 1020 but for SCC samples.

iii) DFs that also respond to some histologic patterns that are not diagnostically relevant for ADC or SCC but may help the network to make a decision. For instance, DF 676, DF 740, and DF 1012 also have high values for tissue patches that contain central airway tissue, which is generally suggestive of SCC as it is often centrally located.

\section{External Validation}

For external validation, 23 lung needle biopsy WSIs were obtained from Grand River Hospital (Kitchener, ON, Canada). These WSIs were composed of $12 \mathrm{SCC}$ and 11 ADC cases. KimiaNet's pipeline was applied to each WSI to 


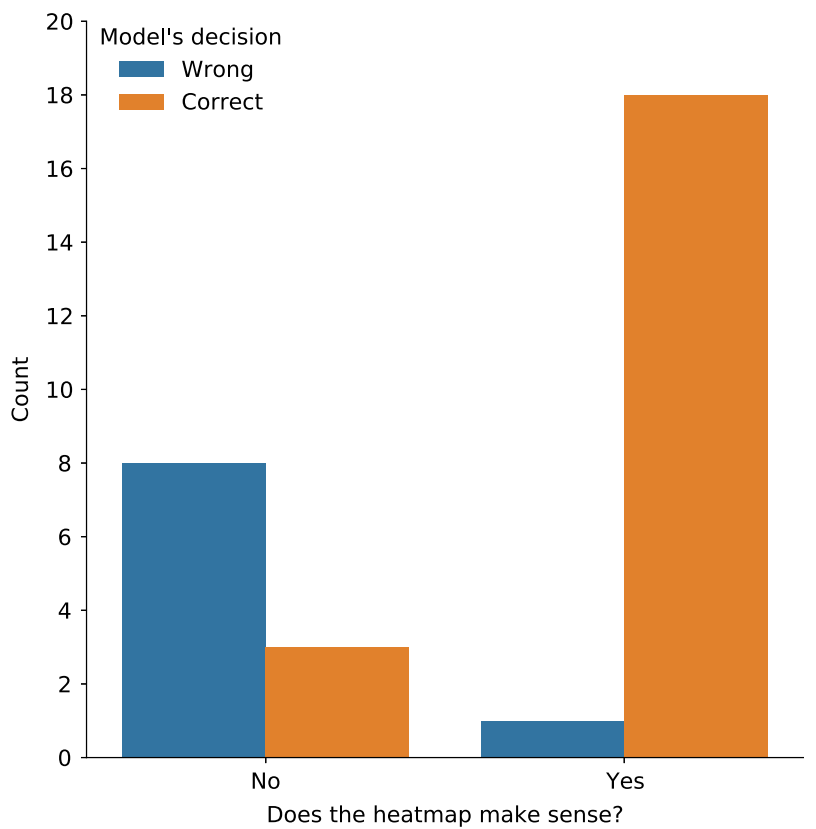

Figure 6 Tissue patch-level heatmaps were examined by the pathologists to determine if they correspond to malignant regions of tissue patches. Next, they were asked whether the model's decision was correct or wrong. One could see that the likelihood of the model's decision is significantly higher when the heatmaps are histopathologically sensible. Consequently, such heatmap visualizations could help pathologists to decide how reliable the output of a model is.

extract deep features from high cellular tissue patches, and MDF values were calculated afterward. Most models trained on single DF values showed significant decreases in performance, except for DF 929 and DF 1005, which somehow retained their classification capabilities. Detailed results are shown in Table 3. However, this issue was attributable to the generalizability of deep learning models as the classification accuracy decreased to $56 \%$ performance even when all 1024 DFs were used. The accuracy was $86 \%$ when applied on KimiaNet's test set.

\section{Discussion}

The interpretability of AI algorithms is defined as the possibility of drawing cause-and-effect relationships from a data-driven model's inputs and outputs. A model is interpretable if a user can predict its output given a set of inputs. ${ }^{37}$ This study simulated the learning process of a deep network (namely, KimiaNet) that has been trained for cancer subtype classification on the loosely labeled data of TCGA data set to be ultimately used for feature extraction. The ultimate goal was to shed light on what this particular network had learned and how it could be utilized to use the learned concepts to improve the interpretability of deep features. The median of deep feature (MDF) vectors of tissue patches extracted from high cellular regions of WSIs were used to simplify the simulation of the learning process. The MDF vectors of tissue patches of size $1000 \times 1000$ pixels could succinctly represent lung cancer WSIs. This approach made it easier to analyze each deep feature independently. Using the MDF vectors could also simplify WSI representation in content-based image retrieval systems. For more heterogeneous specimens, perhaps a cluster-based MDF would be a more suitable approach.

Investigation of deep features indicated that there were 15 features that could single handedly distinguish lung ADC WSIs from lung SCC WSIs with $>85 \%$ accuracy. This is a surprising finding, indicating that it is possible to accurately classify lung ADC and SSC by only assigning one number to a WSI. A single number can determine whether the tumor

Table 3 Lung ADC Versus SCC Classification Using Only One Deep Feature on External Validation Data

\begin{tabular}{|c|c|c|c|c|c|c|}
\hline Case no. & Deep feature & Accuracy & Precision $A D C$ & Precision SCC & Recall ADC & Recall SCC \\
\hline 1 & DF 632 & 0.57 & 0.54 & 0.60 & 0.64 & 0.50 \\
\hline 2 & DF 740 & 0.52 & 0.50 & 0.53 & 0.36 & 0.67 \\
\hline 3 & DF 1020 & 0.39 & 0.41 & 0.33 & 0.64 & 0.17 \\
\hline 5 & DF 641 & 0.52 & 0.00 & 0.52 & 0.00 & 1.00 \\
\hline 6 & DF 1001 & 0.39 & 0.40 & 0.38 & 0.55 & 0.25 \\
\hline 9 & DF 676 & 0.65 & 1.00 & 0.60 & 0.27 & 1.00 \\
\hline 10 & DF 550 & 0.57 & 1.00 & 0.55 & 0.09 & 1.00 \\
\hline 11 & DF 1005 & 0.74 & 0.69 & 0.80 & 0.82 & 0.67 \\
\hline 12 & DF 620 & 0.39 & 0.42 & 0.25 & 0.73 & 0.08 \\
\hline 13 & DF 929 & 0.83 & 0.77 & 0.90 & 0.91 & 0.75 \\
\hline 14 & DF 1007 & 0.52 & 0.00 & 0.52 & 0.00 & 1.00 \\
\hline
\end{tabular}

The table shows that most DFs lost their performance when applied to external validation data. However, DF 929 achieved similar accuracy to that of The Cancer Genome Atlas images. DF 1005 also retained its capability to a lesser extent. Highest values are marked in bold.

ADC, adenocarcinoma; DF, deep feature; SCC, squamous cell carcinoma. 
is lung ADC or SCC. This could make classification more interpretable as one parameter is much easier to interpret than a vector of 1024 real-valued numbers. Consequently, it would be plausible to adjust the threshold value for a single number to modify the model's sensitivity and specificity, making classification more suitable for clinical utility. Median of a single deep feature vector improves the model's interpretability as it is easier to examine a single value in contrast to a vector of 1024 numbers. In addition, although most prominent DFs were not generalizable to the external validation data, two of these DFs (DF 1005 and DF 929) significantly outperformed using all 1024 DFs combined. This finding suggests that reducing the length of DF vectors could potentially improve the generalizability of deep models.

Deep feature heatmaps showed that prominent features are histologically relevant. It was also possible to investigate correlations of prominent deep features with histopathologic patterns. However, a cause-and-effect relationship was not established between deep features on one side and histopathologic patterns on the other side. The overlapping heatmap approach could help investigate deep features at the WSI level with higher resolution. Therefore, instead of looking within an input image, one can investigate at lower magnification. In addition, because these deep features are the results of average pooling from the last convolutional layer of KimiaNet, it was possible to directly visualize each filter's output. The main benefit of using this approach is that it can be done without the need to run another model to generate activation maps. This could be supplementary to attention visualization models that localize regions of importance of a given input image. ${ }^{31}$ These models struggle on images with no apparent region of importance, which is common in high-magnification histopathology images. Furthermore, these tissue patch-level heatmaps could help pathologists to evaluate the reliability of the model's decision. Subjective evaluation showed that such visualizations may display the rationale behind the model's decision, which could help pathologists to evaluate the reliability of a decision made by deep models. Within the context of machine learning and AI, explainability refers to the transparency levels of inherent functionalities of a model to be explained in human terms. ${ }^{38}$ For instance, deep features extracted from histopathologic images must be attributed to well-known histologic patterns so that they can be explained comprehensibly to pathologists. If these deep features are used in an image retrieval system, their explainability should result in image retrieval explainability, a desirable performance improvement that would immensely increase the utility of image search. Consequently, similarities in deep features could be translated into histopathologic similarities. For a diagnosis based on the retrieval of similar results, it would not be achievable unless physician end users understand the rationale behind similarity matching. In this study, tissue regions with the highest value for each prominent DF were subjectively investigated. Although some patterns were observable among those patches, most of these DFs were corresponding to ADC or SCC rather than a specific histologic pattern.

Furthermore, prominent deep features derived from lung cancer images could be utilized to distinguish 21 carcinomas of nonpulmonary organs. This finding confirms the possibility of decreasing the length of deep feature vectors to a much smaller number than 1024 without losing discrimination power. The length of deep feature vectors is one of the factors that complicate the interpretability of deep models.

This study conducted investigations of the interpretability of KimiaNet's deep features, a network trained on hypercellular carcinoma images. Results of the study indicate using the median of deep feature (MDF) vectors to efficiently represent gigapixel WSIs, an absolutely necessary condition for an explainability attempt. In addition, some of the MDF values showed strong correlations with either lung SCC or ADC. These prominent deep features could also cluster ADC and SCC of nonpulmonary organs, which could be further investigated by an elaborate feature selection scheme to decrease the length of deep feature vectors from 1024 to a much smaller number. The study also showed that the quality of these prominent deep features found on the WSI level is somehow transferable to the tissue patch level, both within TCGA test set WSIs of KimiaNet. These prominent deep features could also be used for distinguishing noncancerous and cancerous regions, a task for which KimiaNet had not been trained. The fact that a single prominent deep feature could single handedly discriminate lung SCC from ADC was not only surprising, but also opened the door for easier interpretability through heatmaps, dendrograms, and t-distributed stochastic neighbor embedding visualizations. This may have a far-reaching effect on AI solutions for search and classification. For future work, it would be interesting to apply feature selection schemes to find a smaller subset of deep features that could efficiently represent tissue patches and WSIs. Furthermore, it would be necessary to explore prominent deep features to find out with which visual patterns they correspond to . A smaller set of explainable features will make data-driven algorithms, particularly deep networks, much more accessible to medical professionals, such as pathologists, thereby increasing the clinical utility of AI-based software solutions. Because of the depth and complexity of KimiaNet's structure, deep feature maximization techniques did not produce meaningful results to generate patterns attributable to each prominent DF. To provide compelling explanations for prominent deep features, it would be logical to utilize generative adversarial networks to produce synthetic images that maximize the output of a certain deep feature, similar to what Levine et $\mathrm{al}^{39}$ did to synthesize diagnostic quality pathology images but for deep features rather than the model's classification output. 


\section{Supplemental Data}

Supplemental material for this article can be found at http://doi.org/10.1016/j.ajpath.2021.08.013.

\section{References}

1. Pantanowitz L, Evans A, Pfeifer J, Collins L, Valenstein P, Kaplan K, Wilbur D, Colgan T: Review of the current state of whole slide imaging in pathology. J Pathol Inform 2011, 2:36

2. Wu P, Qu H, Yi J, Huang Q, Chen C, Metaxas D: Deep Attentive Feature Learning for Histopathology Image Classification. 2019 IEEE 16th Int Symp Biomed Imaging (ISBI 2019): IEEE, 2019. pp. $1865-1868$

3. Korbar B, Olofson A, Miraflor A, Nicka C, Suriawinata M, Torresani L, Suriawinata A, Hassanpour S: Deep learning for classification of colorectal polyps on whole-slide images. J Pathol Inform 2017, 8:30

4. Araújo T, Aresta G, Castro E, Rouco J, Aguiar P, Eloy C, Polónia A, Campilho A: Classification of breast cancer histology images using convolutional neural networks. PLoS One 2017, 12:e0177544

5. Yousefi S, Nie Y: Transfer Learning from Nucleus Detection to Classification in Histopathology Images. 2019 IEEE 16th Int Symp Biomed Imaging (ISBI 2019): IEEE, 2019. pp. 957-960

6. Cui Y, Zhang G, Liu Z, Xiong Z, Hu J: A deep learning algorithm for one-step contour aware nuclei segmentation of histopathology images. Med Biol Eng Comput 2019, 57:2027-2043

7. Chen H, Qi X, Yu L, Heng P-A: DCAN: Deep Contour-Aware Networks for Accurate Gland Segmentation. 2016 IEEE Conf Comput Vis Pattern Recognit: Venice, Italy: IEEE, 2016. pp. 2487-2496

8. Wang S, Yang DM, Rong R, Zhan X, Xiao G: Pathology image analysis using segmentation deep learning algorithms. Am J Pathol 2019, 189:1686-1698

9. Bel Tde, Hermsen M, Smeets B, Hilbrands L, Laak Jvander, Litjens G: Automatic segmentation of histopathological slides of renal tissue using deep learning Proc. SPIE 10581, Medical Imaging. Digital Pathol 2018:1058112

10. Coudray N, Ocampo PS, Sakellaropoulos T, Narula N, Snuderl M, Fenyö D, Moreira AL, Razavian N, Tsirigos A: Classification and mutation prediction from non-small cell lung cancer histopathology images using deep learning. Nat Med 2018, 24:1559-1567

11. Tabibu S, Vinod PK, Jawahar CV: Pan-renal cell carcinoma classification and survival prediction from histopathology images using deep learning. Sci Rep 2019, 9:10509

12. Kumar MD, Babaie M, Tizhoosh HR: Deep Barcodes for Fast Retrieval of Histopathology Scans. 2018 Int Jt Conf Neural Networks: IEEE, 2018. pp. 1-8

13. Kalra S, Tizhoosh HR, Shah S, Choi C, Damaskinos S, Safarpoor A, Shafiei S, Babaie M, Diamandis P, Campbell CJV, Pantanowitz L: Pancancer diagnostic consensus through searching archival histopathology images using artificial intelligence. NPJ Digit Med 2020, 3:31

14. Hegde N, Hipp JD, Liu Y, Emmert-Buck M, Reif E, Smilkov D Terry M, Cai CJ, Amin MB, Mermel CH, Nelson PQ, Peng LH, Corrado GS, Stumpe MC: Similar image search for histopathology: SMILY. NPJ Digit Med 2019, 2:56

15. Schaumberg AJ, Juarez-Nicanor WC, Choudhury SJ, Pastrián LG, Pritt BS, Prieto Pozuelo M, Sotillo Sánchez R, Ho K, Zahra N Sener BD, Yip S, Xu B, Annavarapu SR, Morini A, Jones KA, Rosado-Orozco K, Mukhopadhyay S, Miguel C, Yang H, Rosen Y, Ali RH, Folaranmi OO, Gardner JM, Rusu C, Stayerman C, Gross J, Suleiman DE, Sirintrapun SJ, Aly M, Fuchs TJ: Interpretable multimodal deep learning for real-time pan-tissue pan-disease pathology search on social media. Mod Pathol 2020, 33:2169-2185
16. Sit C, Srinivasan R, Amlani A, Muthuswamy K, Azam A, Monzon L, Poon DS: Attitudes and perceptions of UK medical students towards artificial intelligence and radiology: a multicentre survey. Insights Imaging 2020, 11:14

17. Aktolun C: Artificial intelligence and radiomics in nuclear medicine: potentials and challenges. Eur J Nucl Med Mol Imaging 2019, 46: $2731-2736$

18. Thompson RF, Valdes G, Fuller CD, Carpenter CM, Morin O, Aneja S, Lindsay WD, Aerts HJWL, Agrimson B, Deville C Rosenthal SA, Yu JB, Thomas CR: Artificial intelligence in radiation oncology: a specialty-wide disruptive transformation? Radiother Oncol 2018, 129:421-426

19. Wiljer D: To the lighthouse: embracing a grand challenge for cancer education in the digital age. J Cancer Educ 2020, 35:425-427

20. Long D, Magerko B: What Is AI Literacy? Competencies and Design Considerations. Proc 2020 CHI Conf Hum Factors Comput Syst: New York, NY: ACM, 2020. pp. 1-16

21. Tomczak K, Czerwińska P, Wiznerowicz M: Review The Cancer Genome Atlas (TCGA): an immeasurable source of knowledge. Współczesna Onkol 2015, 1A:68-77

22. Riasatian A, Babaie M, Maleki D, Kalra S, Valipour M, Hemati S, Zaveri M, Safarpoor A, Shafiei S, Afshari M, Rasoolijaberi M, Sikaroudi M, Adnan M, Shah S, Choi C, Damaskinos S, Campbell CJ, Diamandis P, Pantanowitz L, Kashani H, Ghodsi A, Tizhoosh HR: Fine-tuning and training of densenet for histopathology image representation using TCGA diagnostic slides. Med Image Anal 2021, 70:102032

23. Litjens G, Kooi $T$, Bejnordi BE, Setio AAA, Ciompi F, Ghafoorian M, van der Laak JAWM, van Ginneken B, Sánchez CI: A survey on deep learning in medical image analysis. Med Image Anal 2017, 42:60-88

24. van Opbroek A, Ikram MA, Vernooij MW, de Bruijne M: Transfer learning improves supervised image segmentation across imaging protocols. IEEE Trans Med Imaging 2015, 34:1018-1030

25. Christodoulidis S, Anthimopoulos M, Ebner L, Christe A, Mougiakakou S: Multisource transfer learning with convolutional neural networks for lung pattern analysis. IEEE J Biomed Heal Inform 2017, 21:76-84

26. Faust K, Bala S, van Ommeren R, Portante A, Al Qawahmed R, Djuric U, Diamandis P: Intelligent feature engineering and ontological mapping of brain tumour histomorphologies by deep learning. Nat Mach Intell 2019, 1:316-321

27. Faust K, Roohi A, Leon AJ, Leroux E, Dent A, Evans AJ, Pugh TJ, Kalimuthu SN, Djuric U, Diamandis P: Unsupervised resolution of histomorphologic heterogeneity in renal cell carcinoma using a brain tumor-educated neural network. JCO Clin Cancer Inform 2020, 4: $811-821$

28. Huang G, Liu Z, Van Der Maaten L, Weinberger KQ: Densely Connected Convolutional Networks. 2017 IEEE Conf Comput Vis Pattern Recognit: IEEE, 2017. pp. 2261-2269

29. Cortes C, Vapnik V: Support-vector networks. Mach Learn 1995, 20 : 273-297

30. Shaha M, Pawar M: Transfer Learning for Image Classification. 2018 Second Int Conf Electron Commun Aerosp Technol: IEEE, 2018. pp. 656-660

31. Wang W, Shen J: Deep visual attention prediction. IEEE Trans Image Process 2018, 27:2368-2378

32. Wang H, Wang Z, Du M, Yang F, Zhang Z, Ding S, Mardziel P, $\mathrm{Hu}$ X: Score-CAM: Improved Visual Explanations via ScoreWeighted Class Activation Mapping, 2020 IEEE/CVF Conference on Computer Vision and Pattern Recognition Workshops (CVPRW). IEEE, 2020. pp. 111-119

33. Smilkov D, Thorat N, Kim B, Viégas F, Wattenberg M: SmoothGrad: Removing Noise by Adding Noise; 2017. Avaliable at https://arxiv org/abs/1706.03825. Accessed October 18, 2021

34. Selvaraju RR, Cogswell M, Das A, Vedantam R, Parikh D, Batra D Grad-CAM: Visual Explanations from Deep Networks via Gradient- 
Based Localization. 2017 IEEE Int Conf Comput Vis: IEEE, 2017. pp. 618-626

35. Chattopadhay A, Sarkar A, Howlader P, Balasubramanian VN: Grad$\mathrm{CAM}++$ : Generalized Gradient-Based Visual Explanations for Deep Convolutional Networks. 2018 IEEE Winter Conf Appl Comput Vis: IEEE, 2018. pp. 839-847

36. Waskom ML: Seaborn: statistical data visualization. J Open Source Softw 2021, 6:3021

37. Larsson S, Heintz F: Transparency in artificial intelligence. Internet Policy Rev 2020, 9:1-16
38. Holzinger A, Langs G, Denk H, Zatloukal K, Müller H: Causability and explainability of artificial intelligence in medicine. Wiley Interdiscip Rev Data Min Knowl Discov 2019, 9:e1312

39. Levine AB, Peng J, Farnell D, Nursey M, Wang Y, Naso JR, Ren H, Farahani H, Chen C, Chiu D, Talhouk A, Sheffield B, Riazy M, Ip PP, Parra-Herran C, Mills A, Singh N, Tessier-Cloutier B, Salisbury T, Lee J, Salcudean T, Jones SJ, Huntsman DG, Gilks CB, Yip S, Bashashati A: Synthesis of diagnostic quality cancer pathology images by generative adversarial networks. J Pathol 2020, 252: $178-188$ 